\title{
Evaluación de dos variedades de albahaca (Ocimum basilicum L.) cultivadas en Corrientes y del efecto de la aplicación de un bioestimulante natural sobre su productividad.
}

\author{
Burgos, Angela M. ${ }^{1}$; Sottile, Jesica J.'; Domíngez, José F ${ }^{1}$. \\ 'Departamento de Producción Vegetal. Cátedra de Cultivos III. FCA.UNNE. \\ e-mail: burgosangela@agr.unne.edu.ar
}

\begin{abstract}
Resumen
El cultivo de albahaca se condujo bajo un invernadero situado en el Campo Experimental de la Facultad de Ciencias Agrarias (27 $28^{\circ}$ ' 27's, $58^{\circ} 47^{\prime}$ 00'O, 50 m.s.n.m.) de la Universidad Nacional del Nordeste (FCA-UNNE), ubicado al noroeste de la Provincia de Corrientes, Argentina. El material biológico evaluado fueron dos variedades de albahaca conocidas como albahaca de hoja ancha (Ocimum basilicum L. var. genovesa) y albahaca morada (Ocimum basilicum L. var. purpurascens). El objetivo del trabajo fue evaluar el potencial de rendimiento de dos variedades de albahaca cultivadas bajo invernadero y el efecto de la aplicación de Inicuim ${ }^{\circledR}$ sobre la biomasa fresca total y particionada de ambas variedades. En el experimento, la aplicación o no de Inicium $^{\circledR}$ en cada una de las variedades determinó 4 tratamientos que se dispusieron en 3 bloques completos al azar, con 10 plantas en cada una. Se registraron los valores de biomasa fresca, medida como peso fresco individual de cada planta entera $\left(\mathrm{g} \mathrm{pl}^{-1}\right)$ y particionada en hojas $\left(\mathrm{g} \mathrm{pl}^{-1}\right)$, tallos $\left(\mathrm{g} \mathrm{pl}^{-1}\right)$, raíces $\left(\mathrm{g} \mathrm{pl}^{-1}\right)$ e inflorescencias $\left(\mathrm{g} \mathrm{pl}^{-1}\right)$, datos a partir de los cuales se calculó el rendimiento de materia fresca en $\mathrm{t} \mathrm{ha}^{-1}$. El rendimiento promedio obtenido de Ocimum basilicum $L$ var. genovesa $\left(15 \mathrm{t} \mathrm{ha}^{-1}\right)$ prácticamente duplicó al de la var. purpurascens $\left(7,57 \mathrm{t} \mathrm{ha}^{-1}\right)$ expresado en biomasa fresca de la planta entera. La aplicación de Inicium ${ }^{\circledR}$ no produjo aumentos estadísticamente significativos de las variables medidas, respecto a los tratamientos testigo de cada variedad de albahaca.
\end{abstract}

Palabras clave: Ocimum basilicum L. var. genovesa, Ocimum basilicum L. var. purpurascens, rendimiento, albahaca morada, albahaca hoja ancha.

\begin{abstract}
Summary
Basil cultivation was made under greenhouse condition, located in the Experimental Field of the School of Agronomy, National University of the Northeast (FCA-UNNE, its Spanish acronym), in

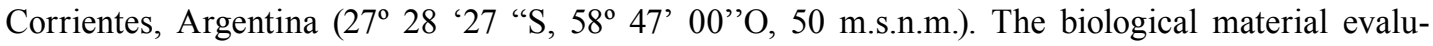
ated were two varieties of basil known as wide leaf basil (Ocimum basilicum L. var. genovese) and purple basil (Ocimum basilicum L. var. purpurascens). The objective was to evaluate the potential performance of two varieties of basil grown under greenhouse condition and the effect of Inicuim ${ }^{\circledR}$ application on total and partitioned fresh biomass in both varieties.In field experiment, the application or non-application of Inicium ${ }^{\circledR}$ on each varieties determined four treatments that were arranged in a randomized complete block with three replications with ten plants. Fresh biomass values, measured as fresh weight of each single whole plant $\left(\mathrm{g} \mathrm{pl}^{-1}\right)$ and sorted in leaves $\left(\mathrm{g} \mathrm{pl}^{-1}\right)$, stems $\left(\mathrm{g} \mathrm{pl}^{-1}\right)$, roots $(\mathrm{g}$ $\left.\mathrm{pl}^{-1}\right)$ and inflorescences $\left(\mathrm{g} \mathrm{pl}^{-1}\right)$ were recorded. Potential yield of Ocimum basilicum L. var. genovese $\left(15 \mathrm{t} \mathrm{ha}^{-1}\right)$ was about double of var. purpurascens $\left(7,57 \mathrm{t} \mathrm{ha}^{-1}\right)$. The application of Inicium ${ }^{\circledR}$ did not showed statistically significant increases on variables measured respect to control treatment in each basil variety.
\end{abstract}

Key words: Ocimum basilicum L. var. genovese, Ocimum basilicum L. var. purpurascens, yield, purple basil, wide leaf basil.

\section{INTRODUCCIÓN}

El género Ocimum está representado por más de 150 especies y tiene una amplia distribución geográfica por todas las regiones de clima tropical y subtropical. Es un importante grupo de plantas aromáticas

Recibido: 14/07/2015. Aceptado: 01/12/2015 que contienen aceites esenciales ricos en diferentes constituyentes, como linalol, geraniol, citral, alcanfor, eugenol, timol, etc. Presenta un inmenso valor para la industria de perfumería y cosméticos, la alimentaria y también la farmacéutica (Sanchez Govin et al., 2000). La albahaca es reconocida por sus propiedades medicinales antisépticas, antiespasmódicas y antiinfla- 
matorias (Sanchez Govin et al., 2000); si bien existen reportados muchos más usos pero no están clínicamente probados. La albahaca (Ocimum basilicum L.) es una planta anual perteneciente a la familia Lamiaceae que abarca una gran cantidad de sub-especies, variedades y formas. Esta especie es consumida mundialmente y en nuestro país se cultiva en los cinturones verdes de las principales ciudades Argentinas y en gran escala en provincia de Buenos Aires, Santa $\mathrm{Fe}$ y Córdoba. El rendimiento de masa verde alcanza el orden de las 20-29 $t$ ha $^{-1}$ año en dos cortes $\left(12 t h^{-1}\right.$ y 8 t ha $^{-1}$ respectivamente) y de $40 \mathrm{Kg} \mathrm{ha}^{-1}$ de aceite esencial (Enciso, 2004). En Corrientes, el rendimiento del cultivo presenta valores superiores a $12 \mathrm{tha}^{-1} \mathrm{de}$ planta fresca, que se elevan a $18 \mathrm{tha}^{-1}$ mediante fertilización nitrogenada (Cenóz y Burgos, 2005), los que se encuentran por encima de los rendimientos promedios a nivel mundial. Por lo expresado, el potencial de producción sería una alternativa altamente favorable a nivel local. Por otra parte, no se tienen registros de producción de determinadas variedades como la denominada albahaca morada (Ocimun basilicum L. var. purpurascens), un híbrido de follaje púrpura (Briseño Ruiz et al.; 2013). Las albahacas de hoja roja/morada, cultivadas para condimento y como ornamentales; son de sabor fuerte, por lo que resultan más atractivas para ensaladas y aderezos, dejan un color rosa en la comida, por lo que se considera ideal para las salsas $\mathrm{y}$ cremas por lo que resultan altamente requeridas en el mercado gourmet. Las albahacas púrpura contienen concentraciones muy altas de antocianos totales y pueden servir como una nueva fuente potencial de pigmentos rojos estables para la industria alimentaria (Simon et al., 1999).

Las semillas de albahaca pueden sembrarse directamente a campo cuando se comercializará deshidratada pero para el consumo en fresco es conveniente producirla en almácigos y en invernaderos, siendo la principal ventaja la uniformidad, vigor y sanidad del cultivo protegido. Previo análisis de suelo, se puede fertilizar ya sea con guano de animales (bien fermentado), o utilizar fertilizantes químicos. Estas aplicaciones pueden realizarse previas a la siembra o plantación en caso del fósforo y dos o tres aplicaciones, durante el ciclo del cultivo para el nitrógeno, con preferencia después de cada corte. Se realizan aplicaciones de materia orgánica y fórmulas completas, cada tres cosechas. Cenóz, Burgos y López (2005), realizaron trabajos en fertilización y concluyeron que el agregado de nitrógeno aumenta significativamente el rendimiento de masa verde total, incrementando el peso y volumen de las plantas; que la relación de palo/hoja aumenta en proporción directa con el aumento de la dosis de nitrógeno; y que el rendimiento de hojas frescas es favorecido con el agregado de nitrógeno aún con la mínima dosis. La últimas tenden- cias en investigación, buscan estrategias de manejo de cultivos que permitan incrementar los rendimientos y cualidades organolépticas a través del uso de productos naturales, compuestos orgánicos, seguros para el ambiente, no tóxicos para humanos ni animales y rápidamente biodegradables, como es el caso del Inicium $^{\circledR}$ (Abdel-Monaim et al., 2014).

En los últimos años se ha creado un nicho de mercado importante para la albahaca, especialmente si es manejado como orgánico, ya que se cree que aumenta sus propiedades medicinales (Murbach-Freire et al., 2005; Fenech et al., 2008). La albahaca orgánica se comercializa a los Estados Unidos de América y a otros países donde prevalece la cultura del uso de alimentos y productos derivados de cultivos orgánicos como parte del sistema de la inocuidad de los alimentos (Ruiz, 2008).

El objetivo del trabajo ha sido evaluar el potencial de rendimiento de dos variedades de albahaca cultivadas bajo invernadero y el efecto de la aplicación de un bioestimulante natural denominado Inicuim ${ }^{\circledR}$, sobre la biomasa fresca total y particionada de ambas variedades.

\section{MATERIALES Y MÉTODOS}

El cultivo de albahaca se condujo bajo un invernadero con forma de arco redondeado y paredes verticales, de estructura metálica y de dimensiones de 25 $\mathrm{m} \times 8 \mathrm{~m} \times 5 \mathrm{~m}$, cubierto con plásticos de 100 y 150 micrones LDT y con media sombra $80 \%$. De paredes verticales con sócalos de $40 \mathrm{~cm}$ de altura, ventilación lateral con cortinas rebatibles en los lados E-O. La ubicación del invernadero y de las lineas dentro del mismo siguió la transecta $\mathrm{N}-\mathrm{S}$.

El invernadero utilizado para el ensayo se encontraba situado en el Campo Didáctico Experimental de la Facultad de Ciencias Agrarias (27 $28^{\circ}$ ' 27''S, $58^{\circ} 47^{\prime} 00^{\prime}$ 'O, 50 m.s.n.m.) de la Universidad Nacional del Nordeste (UNNE), ubicado al noroeste de la Provincia de Corrientes, Argentina.

El suelo del sitio de experimentación ha sido clasificado como Udipsammentes árgico, mixta, hipertérmica, perteneciente a la serie Ensenada Grande (Escobar et al., 1994).

El clima de la región según Köppen corresponde a Cf $w^{*}$ a (h), mesotermal, cálido templado, sin estación seca con precipitaciones máximas en otoño y veranos muy cálidos (Pascale \& Damario, 2004).

El material biológico evaluado fueron dos variedades de albahaca conocidas como Albahaca de hoja ancha (Ocimum basilicum L. var. genovesa) y albahaca morada (Ocimum basilicum L. var. purpurascens) de origen nacional comercializadas por FLORENSA ARG.

Para evaluar el comportamiento de ambas variedades, en primera instancia se trabajó en gabinete 
Tabla 1. Riquezas garantizadas del Inicium ${ }^{\circledR}$ de Bioibérica S.A.

\begin{tabular}{lr}
\hline Materia orgánica & $40,0 \% \mathrm{P} / \mathrm{P}$ \\
Nitrógeno Total $(\mathrm{N})$ & $5,5 \% \mathrm{P} / \mathrm{P}$ \\
Nitrógeno Orgánico & $5,5 \% \mathrm{P} / \mathrm{P}$ \\
Fósforo (P) & $2,4 \% \mathrm{P} / \mathrm{P}$ \\
\hline
\end{tabular}

Tabla 2. Descripción de los tratamientos del experimento llevado a cabo en Corrientes con dos variedades de albahaca.

\begin{tabular}{ll}
\hline Tratamiento 1 & Ocimum basilicum L. var. genovesa sin Inicium \\
Tratamiento 2 & Ocimum basilicum L. var. genovesa con Inicium \\
Tratamiento 3 & Ocimum basilicum L. var. purpurascens sin Inicium \\
Tratamiento 4 & Ocimum basilicum L. var. purpurascens con Inicium \\
\hline
\end{tabular}

donde se midió el poder germinativo (PG) y la energía germinativa (EG), esta última evaluada al 4to día de la siembra. El PG de cada variedad indica la capacidad de las semillas expresado en porcentaje, para poder germinar y dar plántulas normales; mientras que la EG se refiere al porcentaje de semilla en la muestra que ha germinado durante una prueba hasta el momento en que la cantidad de semilla que germina por día ha llegado a su máximo. Para realizar ambas determinaciones se utilizaron 4 cajas de Petri por variedad, que fueron previamente desinfectadas con alcohol metílico embebido en algodón, lavadas y acondicionadas con discos de papel absorbente para posteriormente ser humedecidas con agua destilada. En cada caja de Petri se sembraron 25 semillas. Posteriormente se las tapó, se las rotuló y se las mantuvo con luz y temperatura ambiente.

En segunda instancia se realizó el experimento de campo, para lo cual las semillas de ambas variedades se sembraron en el mes de septiembre en bandejas plásticas multiceldas de 25 celdas cada una, con una capacidad individual de $100 \mathrm{cc}$ por celda. El susbstrato fue el suelo superficial del sitio del ensayo previamente desinfectado con agua calentada a $80^{\circ} \mathrm{C}$. Las bandejas sembradas fueron colocadas bajo malla de media sombra $(80 \%)$. Se trasplantaron cuando tuvieron 4-6 hojas o $10 \mathrm{~cm}$ de alto.

La aplicación o no del bioestimulante Inicium $^{\circledR}$ cuya composición porcentual se presenta en la Tabla 1, en cada una de las variedades determinó 4 tratamientos (Tabla 2) que se dispusieron en bloques completos al azar, con 3 repeticiones, cada una de 10 plantas. Según lo indicado en marbete Inicium ${ }^{\circledR}$, es un producto natural, libre de hormonas, a base de péptidos caracterizados de bajo peso molecular que actúan como activadores de las proteínas del estrés relacionadas con el trasplante, que contribuye a desarrollar un potente aparato radicular (raíz y pelos absorbentes) y adelanta la evolución de los estados iniciales del cultivo.

Dos semanas antes del trasplante, se aplicó Inicium $^{\circledR}$, en una solución de $6 \mathrm{ml}$ por litro de agua a razón de $10 \mathrm{ml}$ por celda de cada bandeja.

El trasplante en el invernadero se realizó en un marco de $50 \times 30 \mathrm{~cm}$, que corresponde a $66.666 \mathrm{pl}$ $\mathrm{ha}^{-1}$. El cultivo se condujo sin limitaciones hídricas ni nutricionales. Para ello se instalaron cintas de riego por goteo con goteros insertos cada $30 \mathrm{~cm}$ que arrojaban un promedio de 2 litros hora ${ }^{-1}$ por lo que se irrigaba durante 90 a 120 minutos aproximadamente para mantener el suelo a capacidad de campo.

Para garantizar la buena nutrición del cultivo, a los 7 días del trasplante también se realizó fertirriego a todas las plantas de los 4 tratamientos con un fertilizante líquido fabricado por Laboratorios Crova bajo el nombre comercial Fertifox ${ }^{\circledR}$, cuya composición química se presenta en la Tabla 3. La dosis aplicada fue de 2,5 cc por litro de agua.

Tabla 3. Composición química del Fertifox.

\begin{tabular}{lcc}
\hline Composición & Cantidad & Unidad \\
\hline Nitrógeno (N) & 14,2 & $\%$ \\
Fósforo (P) & 3,1 & $\%$ \\
Potasio (K) & 4,3 & $\%$ \\
Acido Naftalenacético & 42 & $\mathrm{mg}$ \\
\hline
\end{tabular}

El control de malezas, se realizó mecánicamente efectuando el pase de cultivador entre lineas y manualmente con azada en la línea.

El cultivo se extendió entre septiembre y febrero. Al cosechar (mes de febrero) se muestrearon al azar sistemático y se pesaron las plantas en balanza digital. Los análisis estadísticos se realizaron con los resultados de las variables medidas en los experimentos de campo: biomasa fresca, medida como peso fresco individual de cada planta entera $\left(\mathrm{g} \mathrm{pl}^{-1}\right)$ y particionadas separadamente en hojas $\left(\mathrm{g} \mathrm{pl}^{-1}\right)$, tallos $\left(\mathrm{g} \mathrm{pl}^{-1}\right)$, raíces $\left(\mathrm{g} \mathrm{pl}^{-1}\right)$ e inflorescencias $\left(\mathrm{g} \mathrm{pl}^{-1}\right)$, a partir de estos datos se calculó el rendimiento en $t$ ha $^{-1}$. Con los resultados de la biomasa; ambas variedades se compararon como tratamientos en sí mismos, y para analizar la respuesta de cada una independientemente frente a la aplicación o no de Inicium $^{\circledR}$ se aplicó el test de t de Student. Finalmente la comparación del factorial 2 x 2, de 4 tratamientos se analizó por ANOVA y la separación de medias por test de Duncan $(0,05 \%)$ utilizando el software InfoStat versión 2012.

\section{RESULTADOS}

En laboratorio, la germinación de las semillas colocadas en cajas de Petri, se produjo a temperatura ambiente; de esta manera, la var. genovesa presentó un PG de $88 \%$ y valores de EG de 52,8 \% mientras para la var. purpurascens, los valores fueron de 72 $\%$ y $33,12 \%$ respectivamente (Tabla 4 ). La EG medida en laboratorio, al expresar la capacidad de las semillas de producir plántulas que emerjan en forma rápida y uniforme, en el campo se traduciría en una 
mayor competencia con malezas tempranas. Por lo general, las plántulas que se originan de las semillas que germinan dentro del período energético constituyen el stock de plantación de mejor calidad.

Tabla 4. Porcentajes de Poder Germinativo (PG) y de Energía Germinativa (EG) de las variedades de albahaca genovesa y purpurascens medidas en laboratorio.

\begin{tabular}{lcc}
\hline Variedad de Albahaca & PG \% & EG \% \\
\hline var. genovesa & 88 & 52,8 \\
var. purpurascens & 72 & 33,12 \\
\hline
\end{tabular}

Por su parte, en el almácigo propiamente dicho, la emergencia de plántulas se inició entre el día 4 y 5.

Por los resultados encontrados (Tabla 4), la var. genovesa presenta mayor PG y EG que la var. purpurascens.

Al analizar particularmente el comportamiento de cada variedad de albahaca se encontró que las medias difirieron entre ambas, siendo significativamente mayor el peso fresco de las plantas enteras y el peso fresco de las hojas en la var. genovesa (Tabla 5).

Tabla 5. Comparación de medias de biomasa fresca de la planta entera y particionada por planta entre variedades de albahaca genovesa y purpurascens, expresados en $\mathrm{g} \mathrm{pl}^{-1}$.

\begin{tabular}{lccc}
\hline Biomasa $\left(\mathrm{g} \mathrm{pl}^{-1}\right.$ ) & var. purpurascens & var. genovesa & p-valor \\
\hline Hoja & 64,67 & 108 & $0,0162^{*}$ \\
Tallo & 49,33 & 72,00 & 0,1230 \\
Inflorescencia & 0 & 4,00 & \\
Raíz & 15,33 & 41,33 & 0,0723 \\
Planta entera & 129,33 & 225,33 & $0,0288^{*}$
\end{tabular}

Medias con $\mathrm{p}<=0,05$ son significativamente diferentes según $t$ de Student.

Bajo estos témninos, al calcular el rendimiento potencial de cada variedad ( $\left.\mathrm{t} \mathrm{ha}^{-1}\right)$ a partir de los resultados de las medias del peso fresco de las plantas enteras (Tabla 5), descontando el de la raíz, se obtuvo que la var. genovesa rindió $15 \mathrm{t} \mathrm{ha}^{-1}$, mientras la var. purpurascens rindió solo $7,57 \mathrm{tha}^{-1}$, duplicando la primera variedad en términos de rendimiento potencial.

En este sentido, Paunero (1997) obtuvo rendimientos de $14,4 \mathrm{t} \mathrm{ha}^{-1}$ de albahaca fresca con almácigo y posterior transplante en cultivos en invernadero en Catamarca, que se corresponderían con los resultados obtenidos en este ensayo. Por su parte, el mismo autor cita que en Salta, los rendimientos de albahaca rubín rojo fueron de $14,9 \mathrm{t} \mathrm{ha}^{-1}$, lo cual duplica- ría el rendimiento alcanzado en Corrientes para esta variedad.

Tabla 6. Respuesta de la albahaca morada (Ocimun basilicum L. var. purpurascens) a los tratamientos sin aplicación (T1) y con aplicación (T2) del fertilizante Inicium ${ }^{\circledR}$ en términos de biomasa fresca de la planta entera y particionada de cada uno de sus órganos, expresados en $\mathrm{g} \mathrm{pl}^{-1}$.

\begin{tabular}{|c|c|c|c|}
\hline \multicolumn{4}{|c|}{ Ocimun basilicum var. purpurascens } \\
\hline Biomasa $\left(\mathrm{g} \mathrm{pl}^{-1}\right)$ & Sin Inicium ${ }^{\circ}(1)$ & Con Inicium (2) & p-valor \\
\hline Hoja & 64,67 & 72 & 0,6166 \\
\hline Tallo & 49,33 & 43,67 & 0,6422 \\
\hline Raíz & 15,33 & 21,33 & 0,4903 \\
\hline Planta Entera & 129,33 & 137 & 0,8235 \\
\hline
\end{tabular}

Medias con $\mathrm{p}<=0,05$ son significativamente diferentes.

Tabla 7. Respuesta de la albahaca hoja ancha (Ocimun basilicum var. genovesa) a los tratamientos sin aplicación (T1) y con aplicación (T2) del fertilizante Inicium $^{\circledR}$ en términos de biomasa fresca de la de planta entera y particionada de cada uno de sus órganos, expresados en $\mathrm{g} \mathrm{pl}^{-1}$.

\begin{tabular}{|c|c|c|c|}
\hline \multicolumn{4}{|c|}{ Ocimun basilicum var. genovesa } \\
\hline Biomasa $\left(\mathrm{g} \mathrm{pl}^{-1}\right)$ & Sin Inicium ${ }^{\circ}(1)$ & Con Inicium (2) & p-valor \\
\hline Hoja & 108 & 130 & 0,2096 \\
\hline Tallo & 72 & 76,67 & 0,6735 \\
\hline Raíz & 41,33 & 29,67 & 0,4628 \\
\hline Inflorescencia & 4 & 1,33 & 0,2588 \\
\hline Planta Entera & 225,33 & 237,67 & 0,7776 \\
\hline
\end{tabular}

Medias con $\mathrm{p}<=0,05$ son significativamente diferentes.

Como se muestra en las Tablas 6 y 7, la aplicación de Inicium ${ }^{\circledR}$ no causó diferencias significativas en ninguna de las variables medidas respecto a los tratamientos testigo de ambas variedades de albahaca.

La fase reproductiva, asociada a la presencia de inflorescencias, fue observada solo en la var. genovesa al momento del muestreo (febrero 2014), la cual fue retrasada por el tratamiento $2\left(1,33 \mathrm{~g} \mathrm{pl}^{-1}\right)$ respecto del tratamiento testigo $\left(4 \mathrm{~g} \mathrm{pl}^{-1}\right)$. La floración de la var. purpurascens se observó más tardíamente en plantas que quedaron en el campo después de la fecha de cosecha de este trabajo.

Tabla 8. Respuesta de la biomasa de planta entera, hojas y tallos, de ambas variedades de albahaca en respuesta a los tratamientos sin aplicación y con aplicación del fertilizante Inicium ${ }^{\circledR}$, expresados en $\mathrm{g} \mathrm{pl}^{-1}$.

\begin{tabular}{|c|c|c|c|c|c|}
\hline & & Tratamiento & $\begin{array}{l}\text { Hoja } \\
\left(\mathrm{g} \mathrm{pl}^{-1}\right)\end{array}$ & $\begin{array}{l}\text { Tallo } \\
\left(\mathrm{g} \mathrm{pl}^{-1}\right)\end{array}$ & $\begin{array}{c}\text { Planta Entera } \\
\left(\mathrm{g} \mathrm{pl}^{-1}\right)\end{array}$ \\
\hline \multirow[t]{2}{*}{ Var. purpurascens } & Sin Inicium & 1 & $64,60 a$ & $49,33 a b$ & 129,33 a \\
\hline & Con Inicium & 2 & $72,00 a$ & 43,67 a & $137,00 \mathrm{a}$ \\
\hline \multirow[t]{2}{*}{ Var. genovesa } & Sin Inicium & 3 & $114,50 \mathrm{~b}$ & $75,00 \mathbf{b}$ & 227,75 b \\
\hline & Con Inicium & 4 & $128,00 \mathbf{b}$ & $73,00 \mathbf{b}$ & $237,50 \mathrm{~b}$ \\
\hline CV \% & & & 20,27 & 22,11 & 22,44 \\
\hline
\end{tabular}

Medias con una letra común no son significativamente diferentes $(\mathrm{p}<=0,05)$. 
Los resultados que se presentan en la Tabla 8 ponen en evidencia que las diferencias en peso fresco de la planta entera y de los órganos aéreos (tallos y hojas) responden más al genotipo, que a la aplicación del Inicium $^{\circledR}$. Permitiendo pasar de 8,6 a 9,13 tha ${ }^{1}$ de peso de planta entera con aplicación del fertilizante en la var. purpurascens; y de 15 a 15,8 tha en la var. genovesa, calculados para la densidad de $66.666 \mathrm{pl} \mathrm{ha}^{1}$.

Si bien las plantas de la var. purpurascens poseen significativamente un menor peso por planta que la var. genovesa (Tabla 5); ambas particionan la materia fresca de manera muy similar entre los distintos órganos, con y sin aplicación de Inicium ${ }^{\circledR}$.

Por lo que se pudo apreciar, la aplicación del Inicium $^{\circledR}$ tendió a incrementar la formación de hojas en ambas variedades de albahaca, al tiempo que el peso del tallo no sufrió incrementos, si bien no tuvieron significancia estadística (Tabla 4 y 5 ).

La falta de sensibilidad de las variables medidas en respuesta a la aplicación del Inicium ${ }^{\circledR}$ pudo deberse al amplio lapso transcurrido para tomar la muestra, que correspondió al fin del ciclo (cosecha), por lo que próximos trabajos deberían evaluar la evolución de las variables en el tiempo.

\section{CONCLUSIONES}

Los resultados preliminares hallados para las condiciones agroecológicas de este experimento conducido en Corrientes bajo invernadero plástico, denotan que el potencial de rendimiento de Ocimum basilicum $L$ var. genovesa prácticamente duplica al de la var. purpurascens. Asimismo, si bien la aplicación del Inicium $^{\circledR}$ tendió a incrementar la formación de hojas en ambas variedades de albahaca, al tiempo que el peso del tallo no se modificó, los resultados no tuvieron significancia estadística $(p<0,05)$ por lo que deberán ser validados.

\section{BIBLIOGRAFÍA}

Abdel-Monaim M.F.; Abdel-Gaid, M.A.; Zayan, S.A. 2014. Effectiveness of organic compounds in controlling root rots/ wilts diseases, growth and yield parameters of pepper. Wudpecker J. Agric. Res., 3(4): 081-089.

Briseño-Ruiz, S.E.; Aguilar-García, M.; Villegas-Espinoza, J.A. 2013. El cultivo de la albahaca. Edit. Centro de Investigaciones Biológicas del Noroeste, S.C. La Paz, Baja California Sur, México. 33 p.

Cenoz, P. J.; Burgos, A. M., López, A. E. 2005.- Influencia de la Fertilización Nitrogenada en el Rendimiento de Albahaca (Ocimum basilicum, L.). Revista Horticultura Argentina. Vol 24.n ${ }^{\circ} 56 / 57$ p:18-20
Enciso, A.J. 2004. Producción y comercialización de plantas aromáticas y especies desecadas. http://www.almeriscan.com/ápices/default.htm.27oct.ISO 9001.

Escobar, EH; Ligier, D.; Melgar, M.; Matteio, H.; Vallejos, O. 1994. Mapa de suelos de los Departamentos de Capital, San Cosme e Itatí de la Provincia de Corrientes, Argentina. p. 129. Publicación del Convenio del Instituto Nacional de Tecnología Agropecuaria (INTA-ICA) y Provincia de Corrientes-CFI, Argentina.

Fenech-Larios, L.; Ruíz-Espinoza, F.H.; García-Hernández, J.L.; Murillo-Amador, B.; González-Ocampo, H. A.; Beltrán Morales, F.A.; Fraga-Palomino, H. 2008. Analysis of agronomic variables of Ocimum basilicum L. under alternative tillage systems and standard organic practices. Trop. Subtrop. Agroecosystems 8: 157-163.

Murbach-Freire, C.M.; Marques, M.O.M.; Costa. M. 2005. Effects of seasonal variation on the central nervous system activity of Ocimum gratissimum L. essential oil. J Ethnopharmacology. In press. Available online 21 November 2005.

Pascale, A. y Damario, E. 2004. Bioclimatología Agrícola y Agroclimatología. Editorial Facultad de Agronomía, UBA $.550 \mathrm{pp}$.

Paunero, I. 1999. El cultivo de la albahaca. Disponible en: http://www.biblioteca.org.ar/hautor.asp. Fecha última consulta: 1/07/2015.

Ruiz Espinoza, F.H. 2008. Efectos de dos sistemas de manejos del suelo en la producción de albahaca (Ocimum basilicum L.) en las condiciones de Baja California Sur. Tesis para obtener el grado de Doctor en Ciencias en Manejo Sustentable de los Recursos Naturales de Zonas Áridas y Semiáridas Facultad de Agricultura y Zootecnia de la Universidad Juárez del estado De Durango, México. PP. 109.

Sanchez Govin, E.; Lopez, L.; Fuentes Hernandez, I.M.; Rodriguez Ferrada, C.A. 2000. Estudio farmacognóstico de Ocimum basilicum L. (albahaca blanca). Rev. Cubana Farm[online]. vol.34, n.3, pp. 187-195. ISSN 1561-2988. Fecha última consulta: 1/07/2015

Simon, J.E.; Morales, M.R.; Phippen, W.V.; Vieira, R.F.; Hao, Z. 1999. Basil: A source of aroma compounds and a popular culinary and ornamental herb. p. 499-505. In: J. Janick (ed.), Perspectives on new crops and new uses. ASHS Press, Alexandria, VA. 